\title{
INTEGRAL OPERATORS INVOLVING WHITTAKER FUNCTIONS
}

\author{
by C. NASIM
}

(Received 2 February, 1981)

1. Introduction. We define the integral operators $\mathscr{G}_{\kappa, \mu}^{\nu}$ and $\mathscr{H}_{\kappa, \mu}^{\nu}$ as

$$
\mathscr{G}_{\kappa, \mu}^{\nu} f=\int_{0}^{\infty} g_{\kappa, \mu}(\nu ; x t) f(t) d t, \quad(x>0),
$$

and

$$
\mathscr{H}_{\kappa, \mu}^{\nu} f=\int_{0}^{\infty} h_{\kappa, \mu}(\nu: x t) f(t) d t, \quad(x>0),
$$

where

$$
\begin{aligned}
& g_{\kappa, \mu}(\nu: x)=x^{\nu-1} e^{-\frac{1}{2} x} W_{\kappa, \mu}(x), \\
& h_{\kappa, \mu}(\nu: x)=x^{\nu-1} e^{-\frac{1}{2} x} M_{\kappa, \mu}(x),
\end{aligned}
$$

and $W_{\kappa, \mu}$ and $M_{\kappa, \mu}$ are the Whittaker's confluent hyper-geometric functions. These operators, in their slightly less general form, have been dealt with in [2] and [4]. There the authors have used the fact that these integral operators can be expressed as compositions of the Kober's fractional integral operators and the modified Laplace operator. Then these operators are inverted accordingly. Generally, this type of technique has been very useful for inverting many kinds of integral equations; and a good account of the procedures involved is given [5].

In this paper, we shall regard the operators defined above from a different point of view. We shall consider equations (1.1) and (1.2) as defining integral transforms of the function $f$ with respect to the kernels $g_{\kappa, \mu}$ and $h_{\kappa, \mu}$ respectively. Our main purpose here is to develop a procedure for inverting these integral transforms. To achieve that, we shall first define inversion operators for each of these transforms and then establish algorithms for their inversions. We shall call these inversion operators, linear differential operators of infinite order; cf $[3,7$ (VII, IX)]. At the end we shall give a few examples to illustrate the techniques developed.

2. The difierential operators. It is an easy matter to see that the operator $\theta^{n}$, where $\theta \equiv-x(d / d x)$ and $n$ a positive integer, has the property that $\theta^{n}\left[x^{\alpha}\right]=(-\alpha)^{n} x^{\alpha}$, for some constant $\alpha$. Hence $p_{n}(\theta)$, a polynomial of $n$th degree in $\theta$, gives

$$
p_{n}(\theta)\left[x^{\alpha}\right]=p_{n}(-\alpha) x^{\alpha}
$$

and

$$
p(\theta)\left[x^{\alpha}\right]=p(-\alpha) x^{\alpha}
$$

where $p(s)=\lim _{n \rightarrow \infty} p_{n}(s)$, the limit of a polynomial. Now for some positive $n$, we write

Glasgow Math. J. 24 (1983) 139-148. 
symbolically,

$$
n^{-\theta}=e^{-\theta \ln n}=\lim _{N \rightarrow \infty} \sum_{k=0}^{N} \frac{(-\ln n)^{k}}{k !} \theta^{k}=\lim _{N \rightarrow \infty} q_{N}(\theta),
$$

where $q_{N}(\theta)$ is a polynomial in $\theta$. Then,

$$
n^{-\theta}\left[x^{\alpha}\right]=\lim _{N \rightarrow \infty} q_{N}(\theta)\left[x^{\alpha}\right]=\lim _{N \rightarrow \infty} q_{N}(-\alpha) x^{\alpha}=(n x)^{\alpha} .
$$

With this understanding and using Euler's product for the gamma function, we define the operator $1 / \Gamma(a+\theta)$, symbolically as

$$
\begin{aligned}
\frac{1}{\Gamma(a+\theta)} & =\frac{1}{\Gamma(a)} \lim _{n \rightarrow \infty} n^{-\theta} \prod_{k=1}^{n}\left(1+\frac{\theta}{a-1+k}\right), \quad(R(a)>0), \\
& =\frac{1}{\Gamma(a)} \lim _{n \rightarrow \infty} n^{-\theta} P_{n}(\theta),
\end{aligned}
$$

$P_{n}(\theta)$ being a polynomial in $\theta$. This is an operator of the type defined in [7, IX]. Next we define the operator $\frac{\Gamma(1+a+\theta)}{\Gamma(b+\theta)}$, by

$$
\begin{aligned}
\frac{\Gamma(1+a+\theta)}{\Gamma(b+\theta)} & =\frac{1}{\Gamma(b-a)} \lim _{n \rightarrow \infty} \sum_{k=0}^{n} \frac{(-1)^{k}(\theta+a)(\theta+a-1) \ldots(\theta+a-k)}{k !(b-a+k)} \\
& =\frac{1}{\Gamma(b-a)} \lim _{n \rightarrow \infty} Q_{n+1}(\theta), \text { say. }
\end{aligned}
$$

We shall call $1 / \Gamma(a+\theta)$ and $\Gamma(1+a+\theta) / \Gamma(b+\theta)$, linear differential operators of infinite order, since these operators are expressed as the limit of polynomials in $\theta$. The effect of these operators on a function $x^{-s}$, where $s=\sigma+i t$ with $\sigma, t$ real, is to reproduce it, except for a constant multiplier. That is,

$$
\begin{aligned}
\frac{1}{\Gamma(a+\theta)}\left[x^{-s}\right] & =\frac{1}{\Gamma(a)} \lim _{n \rightarrow \infty} n^{-\theta} P_{n}(\theta)\left[x^{-s}\right] \\
& =\frac{1}{\Gamma(a)} \lim _{n \rightarrow \infty} n^{-s} P_{n}(s) x^{-s}=\frac{1}{\Gamma(a+s)} x^{-s},
\end{aligned}
$$

using (2.1), (2.2) and Euler's product for the gamma function. Also,

$$
\begin{aligned}
\frac{\Gamma(1+a+\theta)}{\Gamma(b+\theta)}\left[x^{-s}\right] & =\frac{1}{\Gamma(b-a)} \lim _{n \rightarrow \infty} Q_{n+1}(\theta)\left[x^{-s}\right] \\
& =\frac{1}{\Gamma(b-a)} \lim _{n \rightarrow \infty} Q_{n+1}(s) \cdot x^{-s}=\frac{\Gamma(1+a+s)}{\Gamma(b+s)} \cdot x^{-s}
\end{aligned}
$$


for $R(a+s)>0$, using (2.4) above and the expansion, given in [8, p. 260],

$$
\frac{\Gamma(x) \Gamma(1+y)}{\Gamma(x+y)}=\sum_{k=0}^{\infty} \frac{(-1)^{k} y(y-1) \ldots(y-k)}{k !(x+k)}, \quad(y>0) .
$$

Now we shall establish differential operators corresponding to the operators $\mathscr{G}_{\kappa, \mu}^{\nu}$ and $\mathscr{H}_{\kappa, \mu}^{v}$. Since

$$
\begin{aligned}
W_{\kappa, \mu}(x) & =O\left(x^{\kappa} e^{-\frac{1}{2} x}\right), \text { as } \quad x \rightarrow \infty, \\
& =O\left(x^{\frac{1}{2}-\mu}\right), \quad \text { as } x \rightarrow 0,
\end{aligned}
$$

it is easy to see that the function $g_{\kappa, \mu}(\nu: x) \in L^{2}(0, \infty)$, whenever $\nu>|\mu|$, and has Mellin transform given by

$$
\begin{aligned}
g_{\kappa, \mu}^{*}(\nu: s) & =\int_{0}^{\infty} g_{\kappa, \mu}(\nu: x) x^{s-1} d x \\
& =\frac{\Gamma\left(\nu-\mu-\frac{1}{2}+s\right) \Gamma\left(\nu+\mu-\frac{1}{2}+s\right)}{\Gamma(\nu-\kappa+s)},
\end{aligned}
$$

belonging to $L^{2}\left(\frac{1}{2}-i \infty, \frac{1}{2}+i \infty\right)$. The differential operator we now consider is

$$
G(\theta)=\frac{1}{\Gamma\left(\nu+\mu-\frac{1}{2}+\theta\right)} \cdot \frac{\Gamma(\nu-\kappa+\theta)}{\Gamma\left(\nu-\mu-\frac{1}{2}+\theta\right)},
$$

where $\theta \equiv-x(d / d x)$. Similarly using the asymptotic expansion of the function $M_{\kappa, \mu}$, one can show that $h_{\kappa, \mu}(\nu: x) \in L^{2}(0, \infty)$, whenever $-\mu<\nu<\kappa+\frac{1}{2}$, and hence its Mellin transform

$$
h_{\kappa, \mu}^{*}(\nu: s)=\frac{\Gamma(1+2 \mu) \Gamma(\kappa-\nu+1-s) \Gamma\left(\mu+\nu-\frac{1}{2}+s\right)}{\Gamma\left(\frac{1}{2}+\mu+\kappa\right) \Gamma\left(\mu-\nu+\frac{3}{2}-s\right)}
$$

belongs to $L^{2}\left(\frac{1}{2}-i \infty, \frac{1}{2}+i \infty\right)$. The differential operator we now consider is

$$
H(\theta)=\frac{\Gamma\left(\frac{1}{2}+\mu+\kappa\right)}{\Gamma(1+2 \mu)} \cdot \frac{1}{\Gamma\left(\mu+\nu-\frac{1}{2}+\theta\right)} \cdot \frac{\Gamma\left(\mu-\nu+\frac{3}{2}-\theta\right)}{\Gamma(\kappa-\nu+1-\theta)} .
$$

Since both operators $G(\theta)$ and $H(\theta)$ are compositions of the operators of the type defined by (2.3) and (2.4), by the successive applications of these operators and using (2.5) and (3.6), the following properties can readily be established.

$$
\begin{aligned}
G(\theta)\left[x^{-s}\right] & =\frac{1}{\Gamma\left(\nu+\mu-\frac{1}{2}+\theta\right)} \cdot \frac{\Gamma(\nu-\kappa+\theta)}{\Gamma\left(\nu-\mu-\frac{1}{2}+\theta\right)}\left[x^{-s}\right] \\
& =\frac{1}{\Gamma\left(\nu+\mu-\frac{1}{2}+s\right)} \cdot \frac{\Gamma(\nu-\kappa+s)}{\Gamma\left(\nu-\mu-\frac{1}{2}+s\right)} \cdot x^{-s} \\
& =\frac{1}{g_{\kappa, \mu}^{*}(\nu: s)} x^{-s}
\end{aligned}
$$


for $R(\nu+\kappa+s)>1, \nu+\mu-\frac{1}{2}>0$, and $s=\sigma+i$, with $\sigma, t$ real. Similarly,

$$
H(\theta)\left[x^{-s}\right]=\frac{1}{h_{\kappa, \mu}^{*}(\nu: s)} x^{-s},
$$

where $R(\mu-\nu-s)>-\frac{1}{2}, \nu+\mu>\frac{1}{2}$, and $s=\sigma+i t$, with $\sigma, t$ real.

\section{The inversion.}

THEOREM 1. If (i) $f \in L^{2}(0, \infty)$, and (ii) $\mathscr{G}_{\kappa, \mu}^{\nu} f=\int_{0}^{\infty} g_{\kappa, \mu}(\nu: x t) f(x) d t$, then

$$
G(\theta)\left[\mathscr{G}_{\kappa, \mu}^{\nu} f\right]=\frac{1}{x} f\left(\frac{1}{x}\right), \text { a.e. } \quad(x>0) .
$$

(at least at points $x$ where $f(x)$ is continuous), and $\nu>\frac{1}{2}-\mu,|\mu|, \kappa+\frac{1}{2}$. (The operator $G(\theta)$ is defined by (2.7) above.)

Proof. Now $f \in L^{2}$, by hypothesis, and the function $g_{\kappa, \mu} \in L^{2}$, as seen above; hence the integral defining the operator $\mathscr{G}_{\kappa, \mu}^{\nu}$ exists and is abolutely convergent. Also the functions $f$ and $g_{\kappa, \mu}$ have Mellin transforms, denoted by $f^{*}$ and $g_{\kappa, \mu}^{*}$ respectively, belonging to $L^{2}\left(\frac{1}{2}-i \infty, \frac{1}{2}+i \infty\right)$. Thus,

$$
\begin{aligned}
\mathscr{G}_{\kappa, \mu}^{\nu} f & =\int_{0}^{\infty} g_{\kappa, \mu}(\nu: x t) f(t) d t \\
& =\frac{1}{2 \pi i} \int_{\frac{1}{2}-i \infty}^{\frac{1}{2}+i \infty} g_{\kappa, \mu}^{*}(\nu: s) f^{*}(1-s) x^{-s} d s,
\end{aligned}
$$

due to the Parseval theorem for $L^{2}$-functions [6]. Also

$$
\begin{aligned}
G(\theta)\left[\mathscr{G}_{\kappa, \mu}^{\nu} f\right] & =G(\theta)\left[\frac{1}{2 \pi i} \int_{\frac{1}{2}-i \infty}^{\frac{1}{2}+i \infty} g_{\kappa, \mu}^{*}(\nu: s) f^{*}(1-s) x^{-s} d s\right] \\
& =\frac{1}{2 \pi i} \int_{\frac{1}{2}-i \infty}^{\frac{1}{2}+i \infty} g_{\kappa, \mu}^{*}(\nu: s) f^{*}(1-s) d s . G(\theta)\left[x^{-s}\right]
\end{aligned}
$$

Now, due to the property (2.9) and simplifying,

$$
\begin{aligned}
G(\theta)\left[\mathscr{G}_{\kappa, \mu}^{\nu} f\right] & =\frac{1}{2 \pi i} \int_{\frac{1}{2}-i \infty}^{\frac{1}{2}+i \infty} f^{*}(1-s) x^{-s} d s \\
& =\frac{1}{x} f\left(\frac{1}{x}\right) \text { a.e. } \quad(x>0),
\end{aligned}
$$

since the Mellin transform of $1 / x f(1 / x)$ exists. It is denoted by $f^{*}(1-s)$.

This gives us the required inversion, provided one can justify bringing the operator $G(\theta)$ inside the integral sign. This amounts to nothing more that differentiating inside the integral sign, which can be justified by making use of the classical result by noting that the resulting integral converges uniformly on every compact subset of the $x$-axis. 
Example 1. Let $f(x)=J_{\eta}(\sqrt{a x}),(a>0)$. Then, by $[\mathbf{1}$, p. 85],

$$
\begin{aligned}
\mathscr{G}_{\kappa, \mu}^{\nu} f= & \int_{0}^{\infty} g_{\kappa, \mu}(\nu: x t) J_{\eta}(\sqrt{a t}) d t \\
= & 2^{-\eta} \frac{\Gamma\left(\frac{1}{2}+\mu+\nu+\frac{1}{2} \eta\right) \Gamma\left(\frac{1}{2}-\mu+\nu+\frac{1}{2} \eta\right)}{\Gamma(\eta+1) \Gamma\left(1-\kappa+\nu+\frac{1}{2} \eta\right)} x^{-\frac{1}{2} \eta-1} a^{\frac{1}{2} \eta} . \\
& \cdot{ }_{2} F_{2}\left(\frac{1}{2}+\mu+\nu+\frac{1}{2} \eta, \frac{1}{2}-\mu+\nu+\frac{1}{2} \eta ; \eta+1,1-\kappa+\nu+\frac{1}{2} \eta ;-\frac{a}{4 x}\right) \\
= & 2^{-\eta} a^{\frac{1}{2} \eta} \sum_{n=0}^{\infty} \frac{\Gamma\left(\frac{1}{2}+\mu+\nu+\frac{1}{2} \eta+n\right)\left(\frac{1}{2}-\mu+\nu+\frac{1}{2} \eta+n\right)}{\Gamma(1+\eta+n) \Gamma\left(1-\kappa+\nu+\frac{1}{2} \eta+n\right) n !} \\
& \left(-\frac{a}{4}\right)^{n} x^{-\left(1+\frac{1}{2} \eta+n\right)} .
\end{aligned}
$$

Now

$$
\begin{aligned}
G(\theta)\left[\mathscr{G}_{\kappa, \mu}^{\nu} f\right]= & 2^{-\eta} a^{\frac{1}{2} \eta} \sum_{n=0}^{\infty} \frac{\Gamma\left(\frac{1}{2}+\mu+\nu+\frac{1}{2} \eta+n\right) \Gamma\left(\frac{1}{2}-\mu+\nu+\frac{1}{2} \eta+n\right)}{\Gamma(1+\eta+n) \Gamma\left(1-\kappa+\nu+\frac{1}{2} \eta+n\right) n !}\left(-\frac{a}{4}\right)^{n} \\
& . G(\theta)\left[x^{-\left(1+\frac{1}{2} \eta+n\right)}\right] .
\end{aligned}
$$

By using the property (2.9), and simplifying, we obtain

$$
\begin{aligned}
G(\theta)\left[\mathscr{G}_{\kappa, \mu}^{\nu} f\right] & =\frac{1}{x} \sum_{n=0}^{\infty}\left(\frac{1}{2} \sqrt{\frac{a}{x}}\right)^{n+2 n} \frac{1}{\Gamma(1+\eta+n) n !} \\
& =\frac{1}{x} J_{\eta}\left(\sqrt{\frac{\alpha}{x}}\right) \equiv \frac{1}{x} f\left(\frac{1}{x}\right),
\end{aligned}
$$

using Theorem 1 , whence $f(x)=J_{\eta}(\sqrt{a x})$, as predicted.

The above theorem establishes the inversion operators for $\mathscr{G}_{\kappa, \mu}^{\nu}$. Now one can develop a simple inversion algorithm for the operator $\mathscr{G}_{\kappa, \mu}^{\nu}$ which works for a special class of functions.

THEOREM 2. If (i) $\mathscr{G}_{\kappa, \mu}^{\nu} f=\int_{0}^{\infty} g_{\kappa, \mu}(\nu: x t) f(t) d t$ exists, $(x>0)$, and (ii) $\mathscr{G}_{\kappa, \mu}^{\nu} f=$ $\sum_{n=0}^{\infty} a_{n} x^{-n-\alpha},(x>r)$, for some constants $r$ and $\alpha$, then

$$
f(x)=\sum_{n=0}^{\infty} a_{n} \frac{\Gamma(\nu-\kappa+\alpha+n) x^{n+\alpha-1}}{\Gamma\left(\nu+\mu-\frac{1}{2}+\alpha+n\right) \Gamma\left(\nu-\mu-\frac{1}{2}+\alpha+n\right)},
$$

$(x>R)$, for some constant $R$.

Proof. We have seen above that one can retrieve the unknown function $f$ from the 
equation in (i), by the application of the operator $G(\theta)$. That is

$$
\begin{aligned}
\frac{1}{x} f\left(\frac{1}{x}\right) & =G(\theta)\left[\mathscr{G}_{\kappa, \mu}^{\nu} f\right] \\
& =G(\theta) \sum_{n=0}^{\infty} a_{n} x^{-n-\alpha} \\
& =\sum_{n=0}^{\infty} a_{n} G(\theta)\left[x^{-n-\alpha}\right] \\
& =\sum_{n=0}^{\infty} a_{n} G(n+\alpha) \cdot x^{-n-\alpha} \\
& =\sum_{n=0}^{\infty} a_{n} \frac{\Gamma(\nu-\kappa+\alpha+n) x^{-n-\alpha}}{\Gamma\left(\nu+\mu-\frac{1}{2}+\alpha+n\right) \Gamma\left(\nu-\mu-\frac{1}{2}+\alpha+n\right)} .
\end{aligned}
$$

The series being power series, the bringing of the operator $G(\theta)$, a linear differential operator, inside the summation sign is then justified. Hence

$$
f(x)=\sum_{n=0}^{\infty} a_{n} \frac{\Gamma(\nu-\kappa+\alpha+n)}{\Gamma\left(\nu+\mu-\frac{1}{2}+\alpha+n\right) \Gamma\left(\nu-\mu-\frac{1}{2}+\alpha+n\right)} x^{n+\alpha-1} .
$$

EXAMPLE 2. We have seen in Example 1, that if $f(x)=J_{\eta}(\sqrt{a x})$, then

$$
\mathscr{G}_{\kappa, \mu}^{\nu} f=\sum_{n=0}^{\infty} a_{n} x^{-n-\alpha}
$$

where

$$
\alpha=\frac{1}{2} \eta+1 \text { and } a_{n}=2^{-\eta} a^{\frac{1}{2} \eta} \frac{\Gamma\left(\frac{1}{2}+\mu+\nu+\frac{1}{2} \eta+n\right) \Gamma\left(\frac{1}{2}-\mu+\nu+\frac{1}{2} \eta+n\right)}{\Gamma(1+\eta+n) \Gamma\left(1-\kappa+\nu+\frac{1}{2} \eta+n\right) n !}\left(\frac{-a}{4}\right)^{n} .
$$

Thus

$$
f(x)=\sum_{n=0}^{\infty} a_{n} \frac{\Gamma(\nu-\kappa+\alpha+n) x^{n+\alpha-1}}{\Gamma\left(\nu+\mu-\frac{1}{2}+\alpha+n\right) \Gamma\left(\nu-\mu-\frac{1}{2}+\alpha+n\right)},
$$

which on substituting the value for $a_{n}$ and $\alpha$, and simplifying gives

$$
f(x)=\sum_{n=0}^{\infty} \frac{(-1)^{n}}{\Gamma(1+\eta+n) n !}\left(\frac{1}{2} \sqrt{a x}\right)^{2 n+\eta}=J_{\eta}(\sqrt{a x}),
$$

as desired.

Many important functions can be derived as special cases of the function $g_{\kappa, \mu}(\nu: x)$. Consequently, we have inversion algorithm of various integral transforms involving these special functions.

EXAMPLE 3. Let $\kappa=0$. Then

$$
g_{0, \mu}(\nu: x)=x^{\nu-1} e^{-\frac{1}{2} x} W_{0, \mu}(x)=\pi^{-\frac{1}{2}} x^{\nu-\frac{1}{2}} e^{-\frac{1}{2} x} K_{\mu}\left(\frac{1}{2} x\right)
$$


Now if $f(x)=x^{\alpha}$, then by $[1$, p. 131$]$,

and

$$
\mathscr{G}_{0, \mu}^{\nu} f=\frac{\Gamma\left(\alpha+\nu+\mu+\frac{1}{2}\right) \Gamma\left(\alpha+\nu-\mu+\frac{1}{2}\right)}{\Gamma(\alpha+\nu+1)} x^{-(\alpha+1)},
$$

$$
G(\theta)\left[\mathscr{G}_{0, \mu}^{\nu} f\right]=\frac{\Gamma(\nu+\theta)}{\Gamma\left(\nu+\mu-\frac{1}{2}+\theta\right) \Gamma\left(\nu-\mu-\frac{1}{2}+\theta\right)}\left[\mathscr{G}_{0, \mu}^{\nu} f\right] .
$$

Using the property (2.9) of the operator $G(\theta)$ and simplifying, the right-hand side becomes

$$
x^{-(\alpha+1)}=\frac{1}{x} f\left(\frac{1}{x}\right)
$$

due to Theorem 1 , whence $f(x)=x^{\alpha}$, as required.

EXAMPLE 4. Let $\kappa=0, \mu=\frac{1}{2}, \nu=1$; then $g_{0, \frac{1}{2}}\left(l ; e^{-x}\right)$ and

$$
\mathscr{G}_{0, \frac{1}{2}}^{1} f=\int_{0}^{\infty} e^{-x t} f(t) d t
$$

giving us the Laplace transform of $f$. The inversion operator in this case in $G(\theta)=1 / \Gamma(\theta)$. If $f(x)=x^{\alpha}$, then

and

$$
\mathscr{G}_{0, \frac{1}{2}}^{1} f=\Gamma(\alpha+1) x^{-(\alpha+1)}
$$

$$
\frac{1}{\Gamma(\theta)}\left[\Gamma(\alpha+1) x^{-(\alpha+1)}\right]=x^{-(\alpha+1)} \equiv \frac{1}{x} f\left(\frac{1}{x}\right),
$$

due to theorem 1 ; hence $f(x)=x^{\alpha}$.

EXAMPLE 5. Let $\kappa=-\frac{1}{4}, \mu=\frac{1}{4}, \nu=\frac{3}{4}$; then

$$
g_{-\frac{1}{4,4},}\left(\frac{3}{4}: x\right)=\sqrt{\pi} \operatorname{Erfc}(\sqrt{x}) \text {. }
$$

Now if we put $f(x)=e^{-x}$, then $[1$, p. 306],

$$
G_{-\frac{1}{4}, 4}^{\frac{3}{4}} f=\sqrt{\pi}\left(1-\sqrt{\frac{x}{1+x}}\right)=\sum_{n=0}^{\infty} a_{n} x^{-n-\alpha},
$$

where $a_{n}=(-1)^{n} \pi \Gamma(2 n+3) / 2^{2 n+2}[\Gamma(n+2)]^{2}$ and $\alpha=1$. Now according to Theorem 2 ,

as required.

$$
f(x)=\sum_{n=0}^{\infty} a_{n} \frac{\Gamma(n+2)}{\Gamma(n+1) \Gamma\left(n+\frac{3}{2}\right)} x^{n}=\sum_{n=0}^{\infty} \frac{(-1)^{n} x^{n}}{n !}=e^{-x}
$$

Similarly the inversion algorithm for the integral operator $\mathscr{H}_{\kappa, \mu}^{\nu}$, defined in (1.2), can be established using the inversion operator $H(\theta)$ defined in (2.8). We shall simply state the results, which can be proved on the lines parallel to those of Theorems 1 and 2 .

THEOREM 3. If (i) $f \in L^{2}(0, \infty)$ and (ii) $\mathscr{H}_{\kappa, \mu}^{\nu} f=\int_{0}^{\infty} h_{\kappa, \mu}(\nu: x t) f(t) d t,(x>0)$, then

$$
H(\theta)\left[\mathscr{H}_{\kappa, \mu}^{v} f\right]=\frac{1}{x} f\left(\frac{1}{x}\right) \quad(x>0) \quad \text { a.e. }
$$

where $H(\theta)$ is defined by (2.8), with $\mu>|\nu|, \frac{1}{2}-\nu$, and $\nu<\kappa+\frac{1}{2}$. 
EXAmple 6. Let $f(x)=J_{\eta}(\sqrt{x})$. Then, by $[\mathbf{1}$, p. 83],

and

$$
\begin{aligned}
\mathscr{H}_{\kappa, \mu}^{\nu} f & =\int_{0}^{\infty} h_{\kappa, \mu}(\nu: x t) J_{\eta}(\sqrt{x t}) d t \\
& =2^{2 \nu} \frac{\Gamma(2 \mu+1)}{\Gamma\left(\mu+\kappa+\frac{1}{2}\right)} x^{\nu-1} G_{23}^{21}\left(\left.\frac{1}{4 x}\right|_{\nu+\frac{1}{2} \eta, \kappa, \nu-\frac{1}{2} \eta} ^{\frac{1}{2}-\mu, \frac{1}{2}+\mu}\right),
\end{aligned}
$$

$$
H(\theta)\left[\mathscr{H}_{\kappa, \mu}^{\nu} f\right]=\frac{2^{2 \nu} \Gamma(2 \mu+1)}{\Gamma\left(\mu+\kappa+\frac{1}{2}\right)} H(\theta)\left[x^{\nu-1} G_{23}^{21}\left(\left.\frac{1}{4 x}\right|_{\nu+\frac{1}{2} \eta, \kappa, \beta-\frac{1}{2} \eta} ^{\frac{1}{2}-\mu, \frac{1}{2}+\mu}\right)\right] .
$$

Now write the $G$-function in terms of Mellin-Barnes type integral, apply the operator $H(\theta)$ inside the integral sign and simplify, to obtain

$$
\begin{aligned}
H(\theta)\left[\mathscr{H}_{\kappa, \mu}^{\nu} f\right] & =2^{2 \nu} x^{\nu-1} \frac{1}{2 \pi i} \int_{L} \frac{\Gamma\left(\nu+\frac{1}{2} \eta-s\right)}{\Gamma\left(1-\nu+\frac{1}{2} \eta+s\right)}\left(\frac{1}{4 x}\right)^{s} d s \\
& =2^{2 \nu} x^{\nu-1} G_{0.2}^{1,0}\left(\frac{1}{4 x} \mid \nu+\frac{1}{2} \eta, \nu-\frac{1}{2} \eta\right) \\
& =\frac{1}{x} J_{\eta}\left(\frac{1}{\sqrt{ } x}\right) \equiv \frac{1}{x} f\left(\frac{1}{x}\right),
\end{aligned}
$$

according to Theorem 3 . Thus

$$
f(x)=J_{\eta}(\sqrt{x})
$$

as required.

EXAmPLe 7. Let $f(x)=(1+x)^{-\alpha}$. Then

$$
\begin{aligned}
\mathscr{H}_{\kappa, \mu}^{\nu} f= & \frac{\Gamma(1+2 \mu) \Gamma\left(\frac{1}{2}+\mu+\nu-\alpha\right) \Gamma(\kappa-\nu+\alpha)}{\Gamma\left(\frac{1}{2}+\mu-\nu+\alpha\right) \Gamma\left(\frac{1}{2}+\kappa+\mu\right)} \\
& \times x^{\alpha-1}{ }_{2} F_{2}\left(\alpha, \kappa-\nu+\alpha ; \frac{1}{2}+\mu-\nu+\alpha, \frac{1}{2}-\mu-\nu+\alpha ; x\right) \\
& +\frac{\Gamma\left(\nu+\mu+\frac{1}{2}\right) \Gamma\left(\alpha-\nu-\frac{1}{2}-\mu\right)}{\Gamma(\alpha)} x^{\nu-\frac{1}{2}+\mu} \\
& { }_{2} F_{2}\left(\nu+\mu+\frac{1}{2}, \kappa+\mu+\frac{1}{2} ; 1+2 \mu, \frac{3}{2}+\mu+\nu-\alpha ; x\right), \\
& \left(\nu+\kappa+\frac{1}{2}>0, \text { and } \nu-\alpha-\kappa<0\right) . \\
= & \frac{\Gamma(1+2 \mu) \Gamma\left(\frac{1}{2}+\mu+\nu-\alpha\right) \Gamma\left(\frac{1}{2}-\mu-\nu+\alpha\right)}{\Gamma\left(\frac{1}{2}+\mu+\kappa\right) \Gamma(\alpha)} \\
& \times \sum_{n=0}^{\infty} \frac{\Gamma(n+\alpha)(\kappa-\nu+\alpha+n) x^{n+\alpha-1}}{\Gamma\left(\frac{1}{2}+\mu-\nu+\alpha+n\right) \Gamma\left(\frac{1}{2}-\mu-\nu+\alpha+n\right) n !} \\
& +\frac{\Gamma\left(\alpha-\nu-\frac{1}{2}-\mu\right) \Gamma(1+2 \mu) \Gamma\left(\frac{3}{2}+\mu+\nu-\alpha\right)}{\Gamma(\alpha) \Gamma\left(\kappa+\mu+\frac{1}{2}\right)} \\
& \times \sum_{n=0}^{\infty} \frac{\Gamma\left(\nu+\mu+\frac{1}{2}+n\right) \Gamma\left(\kappa+\mu+\frac{1}{2}+n\right)}{\Gamma(1+2 \mu+n) \Gamma\left(\frac{3}{2}+\mu+\nu-\alpha+n\right) n !} x^{n+\nu-\frac{1}{2}+\mu} .
\end{aligned}
$$


Now

$$
H(\theta)\left[\mathscr{H}_{\kappa, \mu}^{\nu} f\right]=\frac{\Gamma\left(\frac{1}{2}+\mu+\kappa\right)}{\Gamma(1+2 \mu)} \cdot \frac{1}{\Gamma\left(\mu+\nu-\frac{1}{2}+\theta\right)} \cdot \frac{\Gamma\left(\mu-\nu+\frac{3}{2}-\theta\right)}{\Gamma(\kappa-\nu+1-\theta)}\left[\mathscr{H}_{\kappa, \mu}^{\nu} f\right] .
$$

Next, writing $\mathscr{H}_{\kappa, \mu}^{\nu} f$ in series expansion, bringing the operator $H(\theta)$ inside the summation notation using the property (2.10) and simplifying, we eventually obtain

$$
\begin{aligned}
H(\theta)\left[\mathscr{H}_{\kappa, \mu}^{\nu} f\right]= & \frac{\Gamma\left(\frac{1}{2}+\mu+\nu-\alpha\right) \Gamma\left(\frac{1}{2}-\mu-\nu+\alpha\right)}{\Gamma(\alpha)} \\
& \times \sum_{n=0}^{\infty} \frac{\Gamma(n+\alpha) x^{n+\alpha-1}}{\Gamma\left(\frac{1}{2}-\nu-\mu+\alpha+n\right) \Gamma\left(\frac{1}{2}+\nu+\mu-\alpha-n\right) n !} \\
& +\sum_{n=0}^{\infty} \frac{C_{n}}{\Gamma(-n)} x^{n+\nu-\frac{1}{2}+\mu} .
\end{aligned}
$$

The second series vanishes for all $n=0,1,2, \ldots$ and using the functional relation $\Gamma(z) \Gamma(1-z)=\pi \operatorname{cosec} \pi z$, the first series gives

$$
\begin{aligned}
H(\theta)\left[\mathscr{H}_{\kappa, \mu}^{\nu} f\right] & =x^{\alpha-1} \sum_{n=0}^{\infty} \frac{\Gamma(n+\alpha)}{\Gamma(\alpha) n !}(-1)^{n} x^{n} \\
& =\frac{x^{\alpha-1}}{(1+x)^{\alpha}} \equiv \frac{1}{x} f\left(\frac{1}{x}\right)
\end{aligned}
$$

according to Theorem 3 . Hence $f(x)=1 /(1+x)^{\alpha}$.

THEOREM 4. If

$$
\begin{aligned}
& \text { (i) } \mathscr{H}_{\kappa, \mu}^{\nu} f=\int_{0}^{\infty} h_{\kappa, \mu}(\nu: x t) f(t) d t, \quad(x>0), \\
& \text { (ii) } \mathscr{H}_{\kappa, \mu}^{\nu} f=\sum_{n=0}^{\infty} a_{n} x^{n-\delta}, \quad(x>r),
\end{aligned}
$$

for some constants $r$ and $\delta$, then

$$
f(x)=\frac{\Gamma\left(\frac{1}{2}+\mu+k\right)}{\Gamma(1+2 \mu)} \sum_{n=0}^{\infty} a_{n} \frac{\Gamma\left(\mu-\nu+\frac{3}{2}+n-\delta\right) x^{\delta-n-1}}{\Gamma(\kappa-\nu+1+n-\delta) \Gamma\left(\mu+\nu-\frac{1}{2}-n+\delta\right)},
$$

$(x>R)$, for some constant $R$.

Example 8. Let $f(x)=(1+x)^{-\alpha}$. Then from Example 7, we have

$$
\mathscr{H}_{\kappa, \mu}^{v} f=\sum_{n=0}^{\infty} a_{n} x^{n-\delta}+\sum_{n=0}^{\infty} a_{n}^{\prime} x^{n-\delta^{\prime}}
$$

where

$$
\begin{aligned}
& a_{n}=\frac{\Gamma(1+2 \mu) \Gamma\left(\frac{1}{2}+\mu+\nu-\alpha\right) \Gamma\left(\frac{1}{2}-\mu-\nu+\alpha\right) \Gamma(n+\alpha) \Gamma(\kappa-\nu+\alpha+n)}{\Gamma\left(\frac{1}{2}+\mu+\kappa\right) \Gamma(\alpha)\left(\frac{1}{2}+\mu-\nu+\alpha+n\right) \Gamma\left(\frac{1}{2}-\mu-\nu+\alpha+n\right) n !} \\
& a_{n}^{\prime}=\frac{\Gamma\left(\alpha-\nu-\frac{1}{2}-\mu\right) \Gamma(1+2 \mu) \Gamma\left(\frac{3}{2}+\mu+\nu+\alpha\right) \Gamma\left(\nu+\mu+\frac{1}{2}+n\right) \Gamma\left(\kappa+\nu+\frac{1}{2}+n\right)}{\Gamma(\alpha) \Gamma\left(\kappa+\mu+\frac{1}{2}\right) \Gamma(1+2 \mu+n) \Gamma\left(\frac{3}{2}+\mu+\nu-\alpha+n\right) n !} \\
& \delta=1-\alpha \quad \text { and } \quad \delta^{\prime}=\frac{1}{2}-\nu-\mu .
\end{aligned}
$$


Now, according to Theorem 4,

$$
\begin{aligned}
f(x)= & \frac{\Gamma\left(\frac{1}{2}+\mu+\kappa\right)}{\Gamma(1+2 \mu)} \sum_{n=0}^{\infty} a_{n} \frac{\Gamma\left(\mu-\nu+\frac{3}{2}+n-\delta\right) x^{\delta-n-1}}{\Gamma(\kappa-\nu+1+n-\delta) \Gamma\left(\mu+\nu-\frac{1}{2}-n+\delta\right)} \\
& +\frac{\Gamma\left(\frac{1}{2}+\mu+\kappa\right)}{\Gamma(1+2 \mu)} \sum_{n=0}^{\infty} a_{n}^{\prime} \frac{\Gamma\left(\mu-\nu+\frac{3}{2}+n-\delta^{\prime}\right) x^{\delta^{\prime}-n-1}}{\Gamma\left(\kappa-\nu+1+n-\delta^{\prime}\right) \Gamma\left(\mu+\nu-\frac{1}{2}-n+\delta^{\prime}\right)} .
\end{aligned}
$$

Substituting the values of the respective constants and simplifying, we obtain

$$
\begin{gathered}
f(x)=\frac{\Gamma\left(\frac{1}{2}+\mu+\nu-\alpha\right) \Gamma\left(\frac{1}{2}-\mu-\nu+\alpha\right)}{\Gamma(\alpha)} \sum_{n=0}^{\infty} \frac{\Gamma(n+\alpha) x^{-n-\alpha}}{\Gamma\left(\frac{1}{2}-\mu-\nu+\alpha+n\right) \Gamma\left(\frac{1}{2}+\mu+\nu-\alpha-n\right) n !} \\
+\sum_{n=0}^{\infty} \frac{C_{n}}{\Gamma(-n)} x^{-\frac{1}{2}-\mu-\nu-n} .
\end{gathered}
$$

The second series obviously vanishes and the first series, on simplifying, gives

$$
f(x)=\sum_{n=0}^{\infty} \frac{(-1)^{n} \Gamma(n+\alpha)}{\Gamma(\alpha) n !} x^{-n-\alpha}=\frac{x^{-\alpha}}{\left(1+\frac{1}{x}\right)^{a}}=\frac{1}{(1+x)^{a}}
$$

as predicted.

\section{REFERENCES}

1. A. Erdélyi et al., Tables of integral transforms, Vol. II, Batemann Manuscript Project, (McGraw-Hill, 1954).

2. G. H. Habibullah, A note on a pair of integral operators involving Whittaker's functions, Glasgow Math. J. 18 (1977), 99-100.

3. C. Nasim, An inversion formula for a class of integral transforms, J. Math. Anal. Appl. 52 (1975), 525-537.

4. R. K. Saxena, An inversion formula for the Varma transform", Proc. Cambridge Philos. Soc., 62 (1966), 467-471.

5. I. N. Sneddon, Mixed boundary value problems in potential theory, (North-Holland, 1966). 1948).

6. E. C. Titchmarsh, The theory of Fourier integrals, Second Edition (Oxford University Press,

7. D. V. Widder, An introduction to transform theory, (Academic Press, 1971).

8. E. T. Whittaker and G. N. Watson, A course of modern analysis, (Cambridge University Press, 1963).

DePaRtMENT OF MATHEMATICS

The University of Calgary

Calgary, Alberta

CANADA 